\title{
Organizational Agility
}

Abe Harraf, Ph.D., University of Northern Colorado, USA

Isaac Wanasika, Ph.D., University of Northern Colorado, USA

Kaylynn Tate, Research Assistant, University of Northern Colorado, USA

Kaitlyn Talbott, Research Assistant, University of Northern Colorado, USA

\begin{abstract}
The current competitive environment is characterized by high-intensity rivalry in a dynamic and uncertain environment. The ability to respond swiftly and effectively to these changes is a necessity that separates successful organizations from those that flounder. This paper reviews the concept of organizational agility by exploring the current understanding of the concept, relevant literature and a conceptual framework. Potential avenues for further research are explored.
\end{abstract}

Keywords: Agility; Organizational Effectiveness; Sustainability; Adaptability; Change Management

\section{INTRODUCTION}

\author{
"Without exception, all of my biggest mistakes occurred because I moved too slowly." \\ John Chambers, Cisco CEO
}

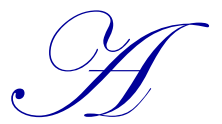

gility in the $21^{\text {st }}$ century is no longer a matter of choice for business organizations. Organizational nimbleness and ability to respond swiftly to the external environment has become a necessity that distinguishes successful organizations from those that flounder. This is compounded by forces of market pressure where successful business practices are mimicked globally. These constant changes require speedy responsiveness and adaptability. However, the inherent necessity to grow and become a contender within respective industries impedes organizations' ability to be flexible and respond to market changes promptly. Sheer size and market power often blinds and hinders the ability to detect and rapidly respond to the changes that could have otherwise improved competitiveness.

There is no quintessential formula for developing an agile firm; a firm can become increasingly agile, but never concretely agile (Alzoubi, 2011). Agility is an ongoing process, much like continuous improvement. Organizational agility is more a matter of becoming than being (Alzoubi, et al., 2011; Doz, 2008; Holsapple \& Li, 2008; Williams \& Lawler, 2013). Organizational agility is considered a core competency, competitive advantage, and differentiator that requires strategic thinking, an innovative mindset, exploitation of change and an unrelenting need to be adaptable and proactive. Agility thus becomes a business imperative for survival rather than choice.

At the basic level, one can break agility into two distinct parts: flexibility and adaptability (Falance, 2012; Holsapple \& Li, 2008). Agility is a measure of responsiveness; anticipated responses to an external stimulus are illustrative of an organization's overall flexibility. The responses and decisions an organization makes in relation to environmental stimuli are a measure of the organization's adaptability. These two responses (effectively planned or unplanned), are primary characteristics of agility that must be constantly enacted by an organization. Breaking agility down into further definitional attributes include an organization's sensing, anticipating, entrepreneurial alertness and proactivity. These elements of agility can be defined as the overarching goals that each pillar of agility is trying to achieve. Conceptualizing dimensions of agility as pillars helps to amplify the foundational and wide scope of agile actions, designed to address specific situations. Pillars of agility are submitted as multiple dimensions of agility that, together, form the core. For example, a pillar emphasizing leadership would present leadership such that it works to make the previously discussed definition of agility possible. 
For an organization to maintain relevance, agility is a priority because it is the essential enabler of organizational effectiveness and excellence (Mische, 2000). It is a means of reaching and sustaining highperformance. Agility must exceed business process and be core characteristic of its people, teams and overall organizational culture.

\section{The State of Agility}

In the recent past, there has been increased activity in trying to comprehend the concept of agility. The changing nature of global competition necessitates a comprehensive understanding of what factors make an organization agile. "We are experiencing not merely another turn of the business cycle, but restructuring of the business order" (Davis, 2009). Globalization has caused markets, technologies, and organizations to be more interconnected. Outsourcing, global partnerships, and overall internationalization all illustrate this shift. Competitive advantage is increasingly becoming temporary due to intensity of global rivalry. Customers expect attractive prices, high quality and fast delivery. Because of the customer-centric nature of agility, in which agility and customer-focus become inherently interrelated, changing customer demands have a significant impact on an organization's operations and must be accounted for when evaluating the external pressures that influence an organization's overall agility (Doz \& Kosonen, 2008).

Beyond the shifting nature of consumer preference, changes in technology contribute to uncertainty, globalized competition and competitive intensity. Digitization facilitates international connections while impacting consumer preferences and the lifecycle of products. Research and development, because of the rapidly changing technological environment, becomes increasingly important to an organization's overall success. Knowledge expansion relates to the speed of adapting to changes. The accessibility of information and ease of information transfer contributes significantly to the need for agility. As knowledge expands, corporations have to respond effectively by sifting through the maze to determine relevant sources of external information and necessary action. Turbulent markets are a new normal that demands swift response by organizations in order to suatain effectiveness.

The adaptation required for an organization to remain relevant is hindered by an organization's age and size. These two principles are foundations of the population ecology (Hannan \& Freeman, 1977) and a significant determinant of the need for agile responses. As companies grow, their size becomes increasingly likely to act as a limiting factor; though as illustrated later this is not a universal truth, organizations must continuously take preventative action. The increasing market pressures for growth, coupled with the potential for rigidity associated with the growth represents a constant contradiction for many organizations. To remedy this potential inconsistency surrounding the importance of growth, efficiency becomes paramount to prevent size from inhibiting and organization's overall ability to respond. As a company grows, it becomes more challenging to have timely responses that span organizational, product and geographic boundaries. Growth is typically associated with how established an organization is. More specifically, as an organization grows, it ages. Like growth, age has the potential to limit an organization's ability to respond accurately to changes in the external environment. Similarly, the scale of operations performed by an organization tends to increase as the organization grows. As a result, mistakes are more likely to be more costly (relative to the cost of errors performed on a smaller organization scale) and plans are often more closely followed to prevent these costly errors. As the consequences associated with erroneous decision-making increases, managers tend to act in a more risk-adverse manner. However, as costsavings becoming more important, agility is often sacrificed (Doz \& Kosonen, 2008). Longevity, growth and cost factors make organizational transformation, response, and overall agility more challenging and less likely to be functionally incorporated into an organization's mindset. Paradoxically, the challenges of remaining agile versus the prominent need for agile responses, is a tension this paper hopes to address by providing an all-encompassing framework. This framework will demonstrate that different organizations have differing needs in terms of agility. Agility is becoming universally important; however, decisions relating to the concept must be made while understanding the organization's unique position in the market (Doz \& Kosonen, 2008).

Agility enables adaptation and response and is increasingly linked to organizational success in today's competitive environment. Speed and responsiveness, two constant components of agility, are critical for competing. However, as previously discussed, they present a challenge for business entities. Because of the changing nature of competition and the inherent volatility of the competitive environment, as well as the fundamental need for an 
organization to more quickly and more accurately respond to the environment, we will outline the components that enable a business to be agile in the shifting landscape. Ultimately, the pillars of agility can be used as a measure for an organization's adaptability, speed, and responsiveness to the evolving external marketplace.

\section{History}

The origin of agility is rooted in the manufacturing industry where adaptability to changes in the supply chain required both flexibility and nimbleness. In the early 1990's, the concept gained recognition among practitioners and researchers in its application to enterprise, where the need for organizational adaptability to change, in the face of dynamic and evolving market conditions, required speed and swiftness.

The history of agility and agile manufacturing stems from that of lean manufacturing (Marchwinski \& Shook, 2007). Indeed, the principles and innovations developed through lean ideologies still directly impact the concept of agility - at times overlapping in both function and objective. In the 1920s, GM introduced mass production and passed Ford as the leading producer of automobiles by pioneering mass production techniques (Marchwinski \& Shook, 2007; Womack \& Jones, 2007). Post World War I, it was largely the automobile industry and this use of mass production that saw the United States become a global economic superpower. However, the competitive advantage the U.S. developed from mass production was easily imitated and in 1955, European manufactures overtook the U.S. manufacturers in both quality and quantity of their outputs. After World War II, lean manufacturing began to develop in response to the shifting market demands associated with the post-war era; a great deal of this effort was concentrated in Japan and specifically in Toyota Motor Corporation. In the 1960s, a well-defined process of lean manufacturing began to form under Eiji Toyoda, Toyota CEO (Womack \& Jones, 2007). Building upon past successes and ideological innovations (including just-in-time inventory management), Toyota Motor Company progressively developed what is today called the Toyota Production System (TPS). TPS is considered a fundamental precursor to lean manufacturing. It married high quality, low costs, short lead-time and an elimination of waste into a system uniquely designed to meet these goals. Crucial to this model's success was Toyota's innovative just-in-time inventory methods and jidoka, automation with a human touch. These two pillars emphasize the manufacturing-centric nature of the TPS model; however, despite this limitation to universal applicability, TPS marks the start of a new innovative production approach. Inclusion of the human element in the manufacturing process was pivotal, and remains applicable in the study of agility today. The interrelationship of processes, quality, and human influence (employees and customers alike) is an underlying concept of TPS, lean manufacturing and, because of the significance of employee involvement and leadership direction, agility. Through the following decades, the system gained recognition and repute. In 1990, researchers from the Massachusetts Institute of Technology published The Machine that Changed the World; a five-year TPS study that concluded the efficiency and effectiveness of TPS was beyond that of traditional mass production (Womack, Jones \& Roos, 1990). As such, it represented a paradigm shift in production ideologies; a shift great enough to warrant the coining of a new term: lean production. The Machine, as the book is colloquially referred, enumerates the advantages of lean manufacturing. This culminates in Toyota's global success: in 2012, Toyota was the world's largest automobile manufacturer and passed General Motors for this spot for the second time since 2007 (Naruo \& Toma, 2007).

As the Toyota Production System began to develop, quality management principles were concurrently being preached throughout Japan. Beginning in 1947, W. Edwards Deming began lecturing Japanese managers on quality control principles. His overall message was that improving quality would lead to a decrease in expenses while increasing the competitive position. This thematic concept explains the rationale for organizational agility: improving an organization's agility will lead to a decrease in expenses while increasing an organization's ability to compete more effectively in a rapidly changing global market. The influence of Deming's work on modern understanding of agility is evident. Total quality management (TQM) and continuous improvement are common components of both lean and agile manufacturing. Both TQM and a commitment to continuous improvement are potential sources of sustainable competitive advantage, and historically significant in the development of agile manufacturing. Features generally associated with TQM transcend the quality boundary and influence an organization's agility (Womack \& Jones, 2007).

As depicted by TPS, Deming, quality management, and other historically significant agility landmarks, the 1990s represented the culmination of lean manufacturing ideologies (Marchwinski \& Shook, 2007). It also marks 
the period in which lean manufacturing was expanded, market dynamics and competitive scale began to change, and agility became a prevailing need for organizations. The two concepts have a complicated relationship though; an organization can be lean, agile, both, or neither. Understanding agility's roots in manufacturing and lean processing facilitates the understanding of its definition and evolution as natural responses to market demands and a continuation of other historical landmarks.

\section{THE PILLARS OF AGILITY}

This section develops a framework of factors that are likely to enhance an organization's agility by evaluating selected research on agility. There is much disparity among scholars as to the attributes and categories of agility. Over this period, scholars have designed a variety of agility frameworks that are similar, and occasionally at odds, with each other. More important, each proposed structure offers new insights into the nature of organizational agility. Our framework of agility is an attempt to provide a simplified, easily applied representation of the most agreed upon aspects of agility. It is designed to prompt a quantifiable measure of an organization's agility, bearing in mind that measures of success are relatively unique to the region, industry, and episodic events. The framework also provides the basis for a tool intended to provide a basic metric for agility. Different categories are identified and described as pillars of agility.

The pillars of agility exist to provide a simplified tool that can be utilized by different organizations, while providing a working framework of agility. The interrelationship between these pillars is obvious though the distinctions between each area can be blurred due to the interrelatedness of the concepts. Though these pillars are presented as individual and isolated components of a relatively abstract concept, connections between the pillars are numerous and indeed, there is no singular or exacting definition of the attributes provided. Instead these pillars exist more as a spectrum within which a firm can perform. Each pillar is defined according to its basic nature as it applies to agility. Subcomponents of each pillar, ideas that exist under the scope of the broader concept, may exist and will similarly be discussed.

\section{Culture of Innovation}

This pillar of agility is foundational as it denotes a specific organizational mindset. An organization with a culture of innovation is one that goes beyond a propensity for change. A culture of innovation implies that an organization is constantly evaluating the systems, structures, procedures, teams, and other organizational components already in place. It is the pressing need to find a new and improved method for performing some function or providing some service (Harraf, 2013). While linked to change management in that a desire for innovation is a spark that stimulates change, a culture of innovation is an underlying, organization-wide look at using changes in the external environment to better shape the organization's internal environment. It is "the ability to do new things and the ability to do old things in new ways" (Holsapple \& $\mathrm{Li}, 2008$ ). It differs from change in that an organization can be innovative without being agile and agile without necessarily being innovative. Despite this, however, there are distinct relationships and some dependencies between innovation, competitiveness, and agility. Innovation, especially in industries of rapid change, is of great importance. More notably, it is a culture of innovation, rather than innovation itself, that is crucial to organizations' success and agility.

\section{Opportunity Seeking and Alertness}

A culture of innovation is one characterized by opportunity-seeking and overall alertness. The organization as a whole must foster an internal desire to discover new opportunities for innovation, be active in actually seeking these out, and act upon the opportunities discovered. Additionally, organizations must be ready to exploit new opportunities by seeking competitive positions in the environment, adapting a posture of openness to new experiences and sustaining creativity. Miles and Snow's prospector strategy (Miles, el al., 1978) most readily aligns with this attribute. The prospector strategy is typically associated with innovation and growing, changing, external environments. Because of this, evaluating an organization according to this standard can prove fruitful. One could argue that, in today's market place, fast-paced, growth, and change, are increasingly becoming the norm (Doz \& Kosonen, 2008). This, in turn, raises both the viability and applicability of the prospector-type strategy in terms of organization's effectiveness and responsiveness. Despite this obvious support for the prospector-type strategy, the 
analyzer typology may still be more applicable for some organizations in certain, more stable, markets. In conjunction with this strategy type, innovation is neither neglected nor prioritized. Instead, innovation happens on the periphery. An analysis of these typologies in relation to opportunity seeking and alertness illuminates several significant points. The culture of innovation has a direct relationship with the external environment as well as the company's overarching strategic objectives. Second, both the prospector and analyzer typologies may successfully foster organizational agility depending on the industry and the relevant external environment. This underscores the notion that agility is not universal or formulaic. It is a "living" concept that adapts to various environments, organizations, and company needs. Evidence suggests that organizations that prioritize innovation, through opportunity seeking and frequently the prospector strategy, are more prepared to successfully utilize additional pillars of agility.

\section{Empowerment}

Empowerment is a pillar that describes the relationship between leadership and employees through authority, autonomy, and other factors. It refers to the degree with which the powers of organizational leaders and lower-level employees are distributed, separated, or shared. The most basic sub-component of this pillar is the concept of centralization and decentralization, and its determination of decision-making authority.

\section{Deference and Decentralization of Power}

Organizations with devolved powers tend to be more agile and better able to respond to the external environment. When lower-level employees have some authority, responses to the environment are faster and more accurate. The caveat to this general rule, however, is that, especially in times of crisis, an upper-level decision is made faster and can be executed more swiftly. This allows for a faster response to an immediate threat or critical problem that allowing lower-level decision-decision makers to respond to would require too much time. Still the trade-off between effectiveness of the response (typically increased by decentralization) and timeliness of the response (often increased by centralization) is such that decentralization often proves more effective. The advantage and effect of lower-level decision making on employees and the organization's responsiveness as a whole results in increased effectiveness and employee morale as a result of autonomy. These advantages outweigh the usual time costs associated with deferring powers to a level significantly below upper management. Thus, this component of agility seems to almost contradict what one might expect: decentralization and deference of decision-making power, rather than centralization, generally increases and organization's overall agility.

\section{Tolerance for Ambiguity}

Agile organizations must thrive in the face of ambiguity. This pillar is similar to the culture of innovation pillar in that it represents an organization's overarching mindset: a culture that transcends organizational levels and boundaries. In the market, especially today, there is an increasing amount of unpredictability. There is not one correct method of responding to the environment, and different responses will be needed at different times. Flexibility is crucial (Falance, 2012; Highsmith, 2010). An organization must be prepared to identify change and respond accordingly. What distinguishes this pillar from market responsiveness is the underlying effect that tolerance for ambiguity has on an organization. Ambiguity is a common nature of the knowledge economy. Although there is abundance of information and knowledge in the marketplace in general, the quantity of information makes finding the relevant data more challenging. Further, competitors tend to have access to similar sources of information, but how they respond is difficult to determine. This adds to the ambiguity associated with global competition. Under uncertain market conditions, an organization meeting the criteria established by this pillar do not stumble or waiver, but thrive in making appropriate decisions in the short-run to meet long-term objectives.

\section{Vision}

An organization's vision is a succinct statement of what an organization daily works to achieve [4]. It is the ultimate guide and unifier as it defines an organization's optimum future state. Its primary functions are to inspire and direct. Within the framework of agility, vision includes not only the written or communicated vision statement, but also the means and methods for establishing and carrying out the organization's vision. This includes 
leadership and other influencers that guide and direct an organization on a business-as-usual scale. These factors aid an organization in aligning with its overall vision. A clearly established vision is crucial to organizational success as it provides managers, employees, governors, etc... an overarching goal to work towards collectively and constantly. Consistency in approach and function increases an organization's agility-and thereby better enable its overall success. As a pillar, an organization's vision has obvious links to other pillars, namely culture of innovation and change management. Vision as it relates to organizational agility is perhaps best summarized by "Simple, clear purpose and principles give rise to complex, intelligent behavior...complex rules and regulations give rise to simple, stupid behavior (Highsmith, 2010)".

\section{Strategic Direction}

An organization's overall direction is established by the vision. However, commitment to the vision and focus on successfully reaching the desired optimum state is critical to its success. Having a vision does not ultimately make an organization any more agile. Having an organizational vision is the norm. Instead, it is communication, adherence to, and focus on achieving the vision that differentiates agile (and typically more successful) organizations from stagnant, less successful ones. Numerous organizations have set forth lofty visions yet faltered in actually seeing these visions come to fruition. The opposite is also true. Organizations that fail often establish visions that are ineffective, uninspiring, or nonexistent. Characteristic of successful visions is a "lasersharp" focus. Having a clear and explicit focus provides an organization with clear strategic direction. Clarity of direction helps an organization respond in an agile and effective manner by establishing the guiding framework for decisions responding to external factors. That is, responding to the external environment is done in a focused manner, in line with the strategic direction a company previously established.

\section{Leadership Roles}

Leadership is the primary purveyor and enforcer of an organization's commitment to its vision. A significant amount of authority and decision making, even in decentralized organizations, stems from leadership (Boaz \& Fox, 2014). Agile companies typically have decision-making occur with three criteria being met: speed, carrying out the actual decision, and rapid response. As previously discussed, speed is often sacrificed for decentralized decision-making. Instead, however, accuracy and employee responsiveness increases. This leads to an overall increase in timeliness of the decision's effective execution. Employees who are more actively involved in decision-making a less likely to be resistant to changes the decision may require. Organizations that can balance decentralization and speed are able to better respond to the external environment and be more agile overall. Further, leadership is ultimately responsible for seeing a decision be carried out. Leaders provide guidance and establish the precedents an organization follows and performs. Rapid response surrounding a decision is related to a leader's ability to communicate a decision and mobilize a response. That is, they can stimulate employee action in a timely manner while minimizing any resistance to a decision that may exist. By achieving this, leaders prime an organization for market responsiveness, thereby establishing an agile precedent from the upper echelons of company hierarchy and down.

Despite the significance of vision, and leadership's role in its effectiveness, the CEO is also responsible for the mundane routine business. Agile organizations know when to respond and equally, when not to respond. There is a propensity for leaders, especially in times of crisis, to overcompensate for an external change. There are situations where leadership must determine that business as usual is the correct response (Glen, 2009). Responding to a change does not always require drastic decisions with substantial effects. Instead, leadership must concern itself with the accuracy of their organization's responses.

\section{Change Management}

Change management has direct linkages with an organization's vision. Change management, by definition, is managing the organization's transition to a desired future state. This can be the transcendent state established by the vision, or a future state that is more limited in scope and smaller in scale. An agile organization is one that can successfully manage change and be aware of the nature of changes occurring in and around itself (Williams, et al., 2013). Change is inevitable. What makes this proposed pillar useful is its assumption that change will come and 
will significantly affect organizations. As with any pillar of agility, change management is flexible and unique to specific organizations. Change will impact different organizations in different ways and the pressures for change an organization faces may be unique to the organization or industry. The nature of change itself, for instance, has evolved dramatically. Change used to be considered a concrete event in which a singular response was enough to mediate the environmental change. Today, however, change is open-ended-an ongoing process that is radical, complex, personal, and continuous (Williams, et al., 2013). Change management is a guide for organizations to become more agile in their navigation of change.

The parallels between change management and decision-making are numerous. Decisions are the essential enablers of change. Organizational decisions lead directly to internal changes when necessary. Beyond this, however, decisions outside of an organization also prompt change. Changes in the external environment may come from consumer decisions and preferences, governmental decisions regarding policy or regulation, or other decisions. This again illustrates the fluid and interrelated nature of agility. One pillar effects another. Likewise, deficits in one area likely pervade others (Boaz \& Fox, 2014).

Change management of agile organizations is comprised of three parts: perceiving the change, implementing change, testing change (Williams, et al., 2013). Perceiving the change pertains to the predisposition of an organization to uncover potential changes, both internal and external. It also highlights the rapidity and accuracy of change perception. Implementing the change is the process of turning a decision into actionable outcomes. Agile organizations see changes implemented faster, smoother, and more accurately than a less agile organization. In order for this to happen, however, managers must be aware of the decision-making (Vision), Team Building, and Organizational Communication pillars. Regarding more agile organizations, this stage of change management requires the organization be able to balance the various components of multiple pillars in accordance to the needs of their organization. Variation in the nature of an organization and the uniqueness of circumstance surrounding change contribute to the disparities companies face. There is no formulaic method to balance the various aspects of change management and the associated pillars of agility to maximize effectiveness. Because of this, implementing change efficiently and effectively, as agile organizations must, is challenging. Once a change is implemented, efficacious change management requires testing. The accuracy and validity of the change should also be reviewed. This review asks, did the change affect the area of the organization where intended? Continuous improvement is enabled through testing change (Williams, et al., 2013). Areas for improvement are discovered, further opportunities highlighted, and knowledge concerning both the internal and external environments is generated such that understanding of both forces is increased.

\section{Communication}

How an organization communicates is also a determinant of its overall agility. This pillar accentuates the importance of communication both within the organization and outside of the organization - with stockholders, potential customers, and other relevant parties in the market. As previous pillars have illustrated, agile organizations must respond to changes, often by themselves also changing. Change facilitates a need for communication, but simultaneously makes effective communication more difficult. This pillar relates quite directly with vision and leadership roles. Decision-making, leadership functions and overall leadership effectiveness are possible only through the various communication channels that exist. That is, while a leader may have an idea or make a decision, it must be communicated throughout an organization and acted upon in order for the decision to be relevant for the organization.

Internal communication responds to the avenues by which information is circulated throughout an organization. There are three primary directions where communication channels exist: top-down, horizontal, and bottom-up. Arguably, the most success and similarly most agile organizations are able to combine an implement these communication methods effectively, facilitating multi-direction and open communication throughout an organization. Top-down communication, communication flowing from upper-level management and down throughout the lower levels of the organization, is perhaps most predisposed to stifling an organization's agility. Crisis may necessitate such limited communication (as the top-down style is constrained by its singularity of direction); however, effective communication most genuinely occurs when the various methods of communication are used in conjunction with one another. Top-down communication was the traditional method of communication 
in many large organizations. However, the changing nature of employees, competition, and the general populous, led to this method of communication's obsolescence. Horizontal and bottom-up communication styles are proven to be more effective in enabling organizational agility. Horizontal communication facilitates interdepartmental relations and exchanges such that repetition or overlap between functions is effectively minimized (Shill, et al., 2012; Yaghoubi \& Dahmardeh, 2010). Encouraging bottom-up communication is similarly crucial in establishing organizational agility through its ability to actively engage all levels of the organization such that resistance to change is minimized, differing opinions are offered, and more effective solutions are potentially formulated insomuch as proximity to some problems is increased.

In addition to formal strategic communication to employees or stakeholders, the firm's informal language as an enabler or constraint for growth and strategic agility is an important dimension of agility (Brannen \& Doz, 2012). Informal communication within the organization facilitates clarity of the firm's strategic position, sensemaking of the external environment and the context of different choices.

\section{Team Building}

A subset of organizational communication that is also related to empowerment is the pillar of team building. Implementation of teams is typically associated with decentralization as teams become more responsible for decision-making and operational success. The tendency of an organization to implement or utilize teams, as well as what the teams look like and how they function, are all influencers of the organization's overall agility. The significance of this pillar is the prioritizing of team activity of specific tasks. . That is, presenting employees with a specific, enumerated task may not result in effective response or task completion. Teams, however, provide a unique element to an organization: unique and diverse perspectives that affect organizational performance, communication, and decision-making. Utilization of teams proves an effective method to better engage employees and reduce resistance to change.

Agile organizations tend to have a propensity for team formation. Like innovation and vision, this aspect of the team-building pillar is representative of an organizational mindset. Teamwork, camaraderie and collaboration should be encouraged on a formal and informal scale. Formalized teams can actively engage in enabling crossdepartmental communication and organizational unity. Creating teams to perform a specified role is an effective method to increase diversity. Glassop (2002) summarizes the advantages of teams as a means of achieving better quality and more thorough and more creative solutions to problems or situations.

While teams may increase the amount of time required to make a decision, the quality of the decision tends to be much higher. Similarly, while conflict may arise in or between different teams, proper implementation of the teams increases organizational unity and positively influences operational outcomes. One pervasive criteria that must consistently be achieved is the organizational premise that teams, not tasks, must be emphasized in order for success to be sustained. To phrase it colloquially, the ends do not justify the means. Though a cliché phrase, the sentiment expressed is particularly applicable to organizations that are dexterous - when successful implementation of some task becomes the primary focus, the costs of achieving the goal increase exponentially. Research suggests that organizations more devoted to the process (namely the employees and teams implemented throughout), produce outcomes more efficiently and more effectively. Investing in the intermediate steps and the process as a whole garners more success than an organization explicitly outcome-oriented and is also characteristic of those organizations who embody the concept of agility.

\section{Market Analysis and Response}

The market analysis and response pillar emphasizes a firm's unique environment surrounding them and their industry as well as the general environment. The overall goal of market responsiveness is to facilitate agility and is actually a measure of both how a firm evaluates its external environment and its propensity to use various tools to do so effectively. More specifically, this pillar provides the tools and metrics for external environment analysis and establishes the need for an organization to use the tools for analysis effectively Williams, et al., 2013). 
Numerous tools exist solely to evaluate the market. While the specific attributes and functions of these tools is not discussed in depth within the context of this paper, preparing an organization for increasingly agile performance requires the implementation of some number of external evaluation tools. Those organizations that expeditiously pursue their external landscape, through the various and numerous means made available to them, prime themselves for further success and nimbleness in regard to adapting and swiftly responding to the external market pressures to which unprepared organizations frequently succumb.

This pillar concurrently seeks to highlight the significance of relationships occurring between members of the same industry. Though paradoxical at the surface, evolving market demands incentivize organizations to build relationships within an industry. Modern research regarding the evolution of market interactions suggests an everincreasing mandate for extra-organizational relationships. According to this assumption, agility is not singularly confined to the internal operations of a single organization. Agility is inherently dependent upon relationships between and among similar companies, even competitors, within an industry. Competition should not be discredited; on the contrary, organizations should be made aware that the nature of competition is undergoing a paradigm shift through which cooperation is progressively more fundamental. This phenomenon is perhaps most adequately summarized, "Competition and cooperation are mutually rooted in and promoted by each other. Cooperation increases the relative scale of market power, resulting in higher intensive competition. Conversely, intensive competition fosters more cooperation between rivals Peng, et al., 2012)". Application of this premise directly to the understanding and extension of agile operations is such that these organizations successfully balance cooperation and competition and achieve competitive harmony within their respective industries that escalates potential market response and thereby furthers opportunities for long-term success and profitability.

\section{Operations Management}

On a broad scale, much of what an organization attempts to fulfil is directly related to either efficiency or effectiveness, and operations management within the context of organizational agility is no exception. Those organizations who consistently behave lithely actively engage in activities particularly crucial to managing efficiency. It is too broad a generalization to conclude all agile organizations are infallibly efficient.

To expand upon some of the organizational capacities that are invariably linked to increasing agility within an organization, one must understand the critical imperative that is continuously seeking to make areas of operation that are not flexible inherently more so. Within the context of supply chain management, for example, is the everrelevant notion that supply chains are increasingly linked and essentially related to one another [9]. That is, the steps integrally required to see a good or service transition from supplier to customer are more dependent upon multiple companies from raw material suppliers to retailers. (It is of significant importance to note that while the supply chains for service organizations may be comprised of steps and processes different from those commonly associated with manufacturing, the principles of supply chain management in fostering organizational responsiveness are the same regardless of the process's output). With these principles considered, the application of them to enabling organizational agility requires only a small extension of what is already well known to operations managers. More specifically, the difference between those organizations who agilely manage their operation, and those who do not, essentially occurs as a result of the flexibility of operations and their consistent and relevant improvement in the face of both ambiguity and intense rivalry.

\section{Structural Fluidity}

Operational design is invariably linked with an organization's ability to behave nimbly within the market. This concept concerns the ways work is initiated, performed, and concluded (Boaz \& Fox, 2014). Organizational structure is the guiding framework that principally drives an organization's performance and establishes the links and communication channels that profoundly influence the organization's vision, fosters flexibility, and creates opportunities for responsiveness. These organizations tend to be flat, without boundaries customer-focused, process oriented, and team based (Dyer, 1998). These characteristics, though distinct in the uniqueness of application to various organizations, are enablers of agility and organizational dexterity. Further, these characteristics demonstrate the structure's function and relationship to other various attributes of agility. Flatter organizations, for example, tend to foster bottom-up and horizontal communication while the lack of structural boundaries enables responsiveness 
and concurrently increases an organization's tolerance for ambiguity. "An organization or systems structure that enables change is based on reusable elements that are reconfigurable in a scalable framework (Highsmith, 2010). Organizational fluidity highlights the significance of flexibility in enabling an organization to be agile. Scholars have further proposed resource fluidity through decoupling, modularizing, dissociating, switching and grafting (Doz \& Kosonen, 2010). These alternatives would facilitate the deployment of new strategic models. Reconfigurable attributes are those that exist on a temporary basis - an organization might use temporary teams to create horizontal linkages between departments to foster the success of a particular project, for example.

\section{Development of a Learning Organization}

The nine pillars of agility previously discussed culminate in the final pillar: development of a learning organization. At the most basic level, a learning organization is one that, through encouraging the learning of its members, seeks to continuously improve and transform, avoiding the potential for stagnancy while improving overall organizational performance. These organizations typically are the most agile, as learning and continuous improvement correlates with responsiveness and embody the notion that every experience an organization faces, good or bad, is an opportunity to learn.

Research defines two methods of learning: single and double loop (Dyer, 1998). Single loop learning seeks to improve through measures and methods previously applied. Continuous improvement can be the focus; however, it is important to clarify: the goals of single-loop learning are seldom questioned and the organization subscribes unwaveringly to specific and enumerated goals. Contrastingly, double-loop learning encourages employees at every organizational level to continuously challenge the practices of the business. This style of learning is more commonly applicable to agile organizations as the need for continuous improvement under this learning style is neither stifled nor constrained by the limitations of the organization's mindset. Ongoing revision becomes the norm as employees seek to revise the organization's overall practices. To define this method of learning explicitly in the context of agility: double-loop learning creates an organizational imperative for responsiveness as internal pressures mount. The organization is not limited in responding to market pressures, but instead must simultaneously achieve internal balance. Organizational goals and outcomes are consistently questioned, demanding the organization either subscribe to its overarching vision, or revise it to better suit the needs of the internal and external environment. A propensity for double-loop learning, however, does not negate the importance of control. Challenging various aspects of the business is intended to drive organizational success and foster improvements, not to actively intend to dismantle infrastructure or negatively evaluate every decision an organization makes.

Developing a learning organization is presented as the final pillar of agility because it provides the necessary tools and cultural implications to enable the previous pillars to exist in a harmonious fashion, unique to the various purposes and functions of differing organizations. By facilitating organizational learning, the provisions and applications of the previous pillars become more readily achievable as fostering a culture concretely devoted to learning and improvement not only enables agility as a broad concept, but also primes an organization to become increasingly responsive through the application of the other various pillars of agility.

\section{CONCLUDING REMARKS}

This paper has developed an organizational agility framework that is grounded on ten pillars. The pillars of agility are: a culture of innovation, empowerment, tolerance or ambiguity, vision, change management, organizational communication, market analysis and response, operations management, structural fluidity and a learning organizations. While the concepts are not new, together they provide a framework for the deployment of an agile organization that is more responsive to the external environment. How the organization goes about employing the measures relating to each attribute should vary according to the needs of the organization itself. Though the application may vary, understanding the degree to which an organization subscribes to the pillars may enable managers to lead their companies towards future success. 


\section{AUTHOR INFORMATON}

Abe Harraf is a professor of management at Monfort College of Business of the University of Northern Colorado. His primary teachings are organizational theory and design and International Management. His research includes organizational performance, agency theory, and economics of firm. He is a recipient of many national and international awards at business and economic conferences and is sought after speaker on management topics. Dr. Harraf currently serves as the chief editor of the Journal of Business and Economic Research (JBER). (Corresponding author)

Isaac Wanasika holds a Ph.D. with a concentration in strategy from New Mexico State University. Prior to that, Isaac held a variety of executive roles in the downstream business of Royal Dutch Shell. His primary teaching area is strategic management. Isaac's research interests include organizational efficiency, transaction cost economics, strategic deception and base-of-the-pyramid strategies. His research has been published in prestigious journals such as the Journal of World Business, Journal of Strategic Innovation and Sustainability, American Journal of Management and the Journal of Managerial Issues. His work has been presented and received awards at national and international business conferences including the Academy of Management. Isaac is currently serving as the Associate Editor for the International Journal of Business and Emerging Markets (IJBEM).

\section{REFERENCES}

Alzoubi, A. E., Al-otoum, F. J., \& Albatainh, A. K. (2011). Factors Associated affecting Organization Agility on Product Development. International Journal of Research and Reviews in Applied Sciences, 9, 503-515.

Becker, B. (2007, October 1). Strategic Agility: The Ultimate Competitive Advantage. Product Development Advantage Group, LLC.,

Brannen, Y. \& Doz, Y. (2012). Corporate languages and strategic agility: trapped in your jargon or lost in translation? California Management Review. 54, 3.

Boaz, N., \& Fox, E. A. (2014). Change leader, change thyself. McKinsey Quarterly. http://mckinsey.com/insights/Leading_in_the_21st_century/...

Daft, R. L. (2011). Organization theory and design (11th ed.). Cincinnati, Ohio: South-Western College.

Davis, I. (2009). The new normal. McKinsey Quarterly, Retrieved from http://www.mckinsey.com/insights/strategy/the_new_normal

Doz, Y., \& Kosonen, M. (2008). Fast Strategy. : Wharton School Publishing.

Dyer, L. R. A. (1998). From human resource strategy to organizational effectiveness: Lessons from research on organizational agility (CAHRS Working Paper \#98-12). Ithaca, NY: Cornell University, School of Industrial and Labor Relations, Center for Advanced Human Resource Studies. http://digital commons.irl.cornell.edu/cahrswp/125

Falance, T. (2012). Cultivating Organizational Agility. The Columbia Group.

Glassop, L. I. (2002). The organizational benefits of teams. Human relations,55 (2), 225-249.

Glenn, M. (2009). Organisational Agility: How business can survive and thrive in turbulent times. The Economist. 125.

Hannan, M. T., \& Freeman, J. (1977). The population ecology of organizations. American journal of sociology, 929964.

Harraf, A. (2013). Strategic Agility: A Required Core Competency in New Normal. 2013 Eurasia Conference. Istanbul, Turkey.

Highsmith, J. (2010). Architects: Accelerators or Anchors to Organizational Agility. SATURN 2010 keynote speech. Software Engineering Institute.

Holsapple, C. and Li, X. (2008). Understanding organizational agility: A work- design perspective. Paper presented at the 13th International Command and Control Research and Technology Symposium. June 17-19, Seattle, WA.

http://www.nps.edu/Academics/ Centers/CEP/docs/2008

Lengnick-Hall and Beck, (2009), C.A. Lengnick-Hall, T.E. Beck

Resilience capacity and strategic agility: Prerequisites for thriving in a dynamic environment. C. Nemeth, E. Hollnagel, S. Dekker (Eds.), Resilience engineering perspectives, 2Ashgate Publishing, Aldershot, UK (2009) 
Marchwinski, C., \& Shook, J. (2007). Lean Lexicon (3e édition, version 3.1 - F. ed.). Institute Lean france: SaintEtienne.

Miles, R. E., Snow, C. C., Meyer, A. D., \& Coleman, H. J. (1978). Organizational strategy, structure, and process. Academy of management review, 3(3), 546-562.

Mische, M. A. (2000). Operational Excellence \& Organizational Agility. University of Southern California.

Naruo, S., \& Toma, S. G. (2007). From Toyota production system to lean retailing. Lessons from Seven-Eleven Japan. In Advances in Production Management Systems (pp. 387-395). Springer US.

Organizational Agility. (2012). MPI Consulting. Retrieved April 20, 2014, from http://managementperformance.com/organizational-agility/

Peng, T.-J. A., Pike, S., Yang, J. C.-H. and Roos, G. (2012), Is Cooperation with Competitors a Good Idea? An Example in Practice. British Journal of Management, 23: 532-560. doi: 10.1111/j.1467-8551.2011.00781.x

PMI Pulse of the Profession In-Depth Report: Organizational Agility, 2002.

Shill, W., Engel, J. F., Mann, D., \& Schatteman, O. (2012). Corporate Agility: Six ways to make volatility your friend. Outlook: The journal of high-performance business,.

Williams, T., Worley, C. G., \& Lawler III, E. E. (2013, April 15). The Agility Factor. Strategy + Business.

Womack, J. P., \& Jones, D. T. (2007). The machine that changed the world: based on the Massachusetts Institute of Technology 5-million dollar 5-year study on the future of the automobile ( ed.). New York: Rawson Associates.

Womack, J., \& Jones, D. D. Roos (1990). The Machine That Changed the World.

Yaghoubi, N. m., \& Dahmardeh, M. R. (2010). Analytical approach to effective factors on organizational agility. Journal of Basic and Applied Scientific Research, 1(1), 76-87. 